\title{
Research on the Development Law of Smart Phone Screen based on User Experience
}

\author{
Pei Xuesheng, ${ }^{1,2}$, , Wang Yang ${ }^{2}$ \\ ${ }^{1}$ School of Mechatronic Engineering and Automation, Shanghai University, Shanghai, China \\ ${ }^{2}$ School of Art and Design, Henan University of Science and Technology, Luoyang, China
}

\begin{abstract}
Modern electronic products are changing with each passing day, and the most prominent one is the smart phone. Screen is the key part for people to extract and exchange information through mobile phones, and its development is very rapid. This article summarize the development laws of screen size, screen occupation ratio, resolution and shape from the perspective of user experience, and analyze the reasons for its development law. According to the research, the optimal size of the mobile phone screen suitable for the users is summed up and verified through the questionnaire, which provides a reliable basis for the developer of mobile phone to design smart phone screen and improve the satisfaction of the user experience.
\end{abstract}

\section{Introduction}

With the advancement of technology, smart phones have become an essential product in people's lives. The screen technology is also rapidly developing, and the screen of the mobile phone has undergone great changes in materials, modeling, size, and resolution. Mobile developers constantly adjust the screen size and screen occupation ratio to bring better visual experience and interactive experience to users.

But what exactly happened to the evolution of the mobile phone screen, what kind of screen size can most meet the needs and comfort of users, this article summarizes the development law of the screen through data collection and collation, and analyze the reasons for the formation of this rule in order to get the best screen size which is suitable for people. This study will provide a theoretical basis for mobile phone developers to design and improve products, help them grasp the future development direction of mobile screens, and then improve the satisfaction of user experience.

\section{Development status of mobile phone screen}

In recent years, the replacement of mobile phones has largely been related to the improvement of the screen. Companies such as Apple, Samsung, HUAWEI and MI have continued to compete in the screen occupation ratio, big screen and high screen ratio become the goals pursued by big enterprises. But while improving the user experience, there are some problems and hazards.

For the user experience of mobile phone and the development of the screen, user experience refers to people's cognitive impressions and responses to products, systems or services that are used or expected to be used. It is a truly extended and distinct perspective on quality of interactive technology ${ }^{[1]}$.Si Yaqing and others collected the satisfaction factors and dissatisfaction factors in the experience of the smart phone users, and put forward the results of the user experience in terms of emotions, satisfaction, continuous use intentions, etc., and elaborated the user's psychological changes when there is a difference between the user's psychological expectations and the actual use of the feelings ${ }^{[2]}$.Tian Meiling and Zhao Xinjun analyzed the development of mobile phone screen based on TRIZ, and proposed that the mobile phone screen will adapt to the high intelligence requirements of the mobile phone, make its resolution higher, the material is more advanced, and the size is more consistent with ergonomics requirements ${ }^{[3]}$. Wang Songpei and others studied the future development direction of the mobile phone screen and proposed that the mobile phone screen will become more beautiful, durable, stylish and portable ${ }^{[4]}$. Xin Yizhong and others proposed that smart phones with large size screen provide more information display space for users, and also bring difficulties to the target selection operation of single hand operation ${ }^{[5]}$. Karlson and others used experiments to evaluate how the size of the device, the target position, and the direction of movement affect the mobility of the thumb when handheld device interacts with one hand ${ }^{[6]}$.

Therefore, study the trends and laws of mobile phone screen, and analyze the negative impact of excessive mobile phone screen to users, comprehensive data to summarize the best screen size for people to use, which will help mobile phone developers understand user demands, target optimization of improved products,

*Corresponding author: ${ }^{\text {a }}$ Pei Xuesheng: pxsh12@126.com 
improve competitive advantage of brand.

\section{Research on the development of mobile phone screen and its laws}

This study summarizes the indicators and data of smart phone screens of Samsung, Apple, and HUAWEI mobile phone enterprises in recent years, reveal their development rules, and analyze the specific reasons for the formation of laws.

The research object selected Samsung Galaxy S Series, Apple iPhone Series and HUAWEI P Series, including two large-screen series of Samsung Galaxy S+ and iPhone plus. The content of the study is the release year, screen size, screen occupation ratio, resolution, and screen shape. The different series of specific information are shown in Table 1, table 2, table 3, table 4, and table 5.

Table 1. Galaxy S Series

\begin{tabular}{cccccc}
\hline $\begin{array}{c}\text { Mobile phone } \\
\text { models }\end{array}$ & $\begin{array}{c}\text { The release } \\
\text { year/year }\end{array}$ & $\begin{array}{c}\text { Screen } \\
\text { size/Inches }\end{array}$ & $\begin{array}{c}\text { Screen occupation } \\
\text { ratio/\% }\end{array}$ & Resolution & Screen shape \\
\hline Galaxy S & 2010 & 4.0 & 58.1 & $480 \times 800$ & Straight screen \\
Galaxy S2 & 2011 & 4.3 & 61.3 & $480 \times 800$ & Straight screen \\
Galaxy S3 & 2012 & 4.8 & 65.9 & $1280 \times 720$ & Straight screen \\
Galaxy S4 & 2013 & 5.0 & 72.3 & $1920 \times 1080$ & Straight screen \\
Galaxy S5 & 2014 & 5.1 & 70.0 & $1920 \times 1080$ & Straight screen \\
Galaxy S6 & 2015 & 5.1 & 70.9 & $2560 \times 1440$ & Straight screen \\
Galaxy S7 & 2016 & 5.1 & 73.9 & $2560 \times 1440$ & Straight screen \\
Galaxy S8 & 2017 & 5.8 & 84.0 & $2960 \times 1440$ & Hyperboloid \\
Galaxy S9 & 2018 & 5.8 & 89.5 & $2960 \times 1440$ & Hyperboloid
\end{tabular}

Table 2. Galaxy S+ Series

\begin{tabular}{cccccc}
\hline $\begin{array}{c}\text { Mobile phone } \\
\text { models }\end{array}$ & $\begin{array}{c}\text { The release } \\
\text { year/year }\end{array}$ & $\begin{array}{c}\text { Screen } \\
\text { size/Inches }\end{array}$ & $\begin{array}{c}\text { Screen occupation } \\
\text { ratio/\% }\end{array}$ & Resolution & Screen shape \\
\hline Galaxy S6 edge & 2015 & 5.1 & 72.0 & $2560 \times 1440$ & Hyperboloid \\
Galaxy S7 edge & 2016 & 5.5 & 76.1 & $2560 \times 1440$ & Hyperboloid \\
Galaxy S8+ & 2017 & 6.2 & 83.3 & $2960 \times 1440$ & Hyperboloid \\
Galaxy S9+ & 2018 & 6.2 & 83.6 & $2960 \times 1440$ & Hyperboloid \\
\hline
\end{tabular}

Table3. HUAWEI P Series

\begin{tabular}{cccccc}
\hline $\begin{array}{c}\text { Mobile phone } \\
\text { models }\end{array}$ & $\begin{array}{c}\text { The release } \\
\text { year/year }\end{array}$ & $\begin{array}{c}\text { Screen } \\
\text { size/Inches }\end{array}$ & $\begin{array}{c}\text { Screen occupation } \\
\text { ratio/\% }\end{array}$ & Resolution & Screen shape \\
\hline HUAWEI P1 & 2012 & 4.3 & 61.0 & $960 \times 540$ & Straight screen \\
HUAWEI P2 & 2013 & 4.7 & 67.0 & $1280 \times 720$ & Straight screen \\
HUAWEI P6 & 2013 & 4.7 & 70.1 & $1280 \times 720$ & Straight screen \\
HUAWEI P7 & 2014 & 5.0 & 71.6 & $1920 \times 1080$ & Straight screen \\
HUAWEI P8 & 2015 & 5.2 & 78.3 & $1920 \times 1080$ & Straight screen \\
HUAWEI P9 & 2016 & 5.2 & 72.5 & $1920 \times 1080$ & Straight screen \\
HUAWEI P10 & 2017 & 5.4 & 71.2 & $1920 \times 1080$ & Straight screen \\
HUAWEI P20 & 2017 & 5.8 & 80.3 & $2244 \times 1080$ & Straight screen \\
\hline
\end{tabular}

Table4. iPhone Series

\begin{tabular}{cccccc}
\hline $\begin{array}{c}\text { Mobile phone } \\
\text { models }\end{array}$ & $\begin{array}{c}\text { The release } \\
\text { year/year }\end{array}$ & $\begin{array}{c}\text { Screen } \\
\text { size/Inches }\end{array}$ & $\begin{array}{c}\text { Screen occupation } \\
\text { ratio/\% }\end{array}$ & Resolution & Screen shape \\
\hline iPhone 4 & 2010 & 3.5 & 54.0 & $960 \times 640$ & Straight screen \\
iPhone 4s & 2011 & 3.5 & 54.0 & $960 \times 640$ & Straight screen \\
iPhone 5 & 2012 & 4.0 & 60.9 & $1136 \times 640$ & Straight screen \\
iPhone 5s & 2013 & 4.0 & 60.9 & $1136 \times 640$ & Straight screen \\
iPhone 6 & 2014 & 4.7 & 65.8 & $1334 \times 750$ & Straight screen \\
iPhone 6s & 2015 & 4.7 & 65.8 & $1334 \times 750$ & Straight screen \\
iPhone 7 & 2016 & 4.7 & 65.6 & $1334 \times 750$ & Straight screen \\
iPhone 8 & 2017 & 4.7 & 65.4 & $1334 \times 750$ & Straight screen \\
iPhone X & 2017 & 5.8 & 81.2 & $2346 \times 1125$ & Straight screen \\
\hline
\end{tabular}


Table5. iPhone plus Series

\begin{tabular}{cccccc}
\hline $\begin{array}{c}\text { Mobile phone } \\
\text { models }\end{array}$ & $\begin{array}{c}\text { The release } \\
\text { year/year }\end{array}$ & $\begin{array}{c}\text { Screen } \\
\text { size/Inches }\end{array}$ & $\begin{array}{c}\text { Screen occupation } \\
\text { ratio/\% }\end{array}$ & Resolution & Screen shape \\
\hline iPhone 6 plus & 2014 & 5.5 & 67.7 & $1920 \times 1080$ & Straight screen \\
iPhone 6s plus & 2015 & 5.5 & 67.7 & $1920 \times 1080$ & Straight screen \\
iPhone 7 plus & 2016 & 5.5 & 67.7 & $1920 \times 1080$ & Straight screen \\
iPhone 8 plus & 2017 & 5.5 & 67.4 & $1920 \times 1080$ & Straight screen \\
\hline
\end{tabular}

\subsection{Analysis of the overall change of mobile phone screen.}

The screen size, screen occupation ratio, resolution, and screen form of the mobile phone have all undergone great changes. The screen size increases gradually in a certain range and then tends to be stable. The screen occupation ratio continues to increase, and it develops in the direction of a full screen. Screen resolution has also been greatly improved to meet users' requirements for screen quality and color. The screen shape also has a new breakthrough. In 2015, Samsung first launched the hyperboloid screen phone, making the mobile phone more rounded and have a wider viewing angle, and improving the user's touch and visual experience.

In general, with the development of science and technology, the upgrading of mobile phone screens continues to meet the needs of users and brings better experience to users. This article will focus on the mobile phone screen size and screen occupation ratio, analyze the law of change and explore its causes, and then speculate the development trend of the mobile phone screen.

\subsection{Analysis of change law of mobile phone screen size}

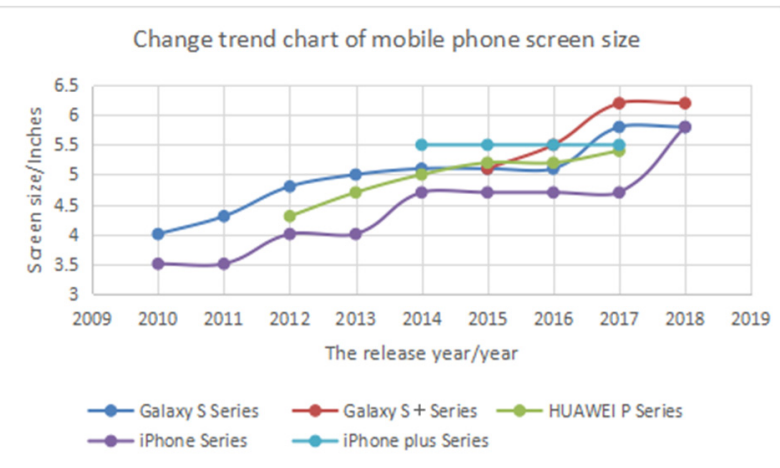

Figure 1. Change trend chart of mobile phone screen size

According to the Figure 1, we can see that since 2010, the size of the mobile phone screens of each brand has been rising, and there are some regularities in the process of rising. It is worth noting that between 2010 and 2014, the overall change in screen size was large and it rose rapidly. However, from 2014 to 2017, the size changes of the Samsung Galaxy S series, the HUAWEI P series, the iPhone series and the iPhone plus series were minimal or even unchanged, showing a basic stable state, with a stable value of 5.1 inches, 5.2 inches, 4.7 inches, and 5.5 inches.

The phenomenon reflects that the screen size of mobile phones will not increase infinitely, the size of the mobile phone screen, and the size of the mobile phone will develop steadily in a suitable area, which is determined by the user's needs and the satisfaction of the user experience.

In order to further confirm the optimal screen size suitable for people's use, this study conducted a single-questionnaire survey on "What size do you think is the most comfortable screen size to use?" The way to answer the questions is to fill in the numbers. The respondents included students, teachers, and the public, among whom 82 were males and 76 were females. Finally, 160 data were retrieved. The effective data was 158. The effective recovery rate was $98.75 \%$, which met the research requirements.

According to statistics, the mean value of data filled by men is 5.30 inches, and the mean value of data filled by women is 5.01 inches. People choose 4.7 inches, 5.5 inches, and 5.1 inches for the best screen size of mobile phone, which is in complete agreement the stable value of three surveyed brands above and verified the value range of the best screen size of mobile phone.

\subsection{Analysis of change law of mobile phone screen occupation ratio}

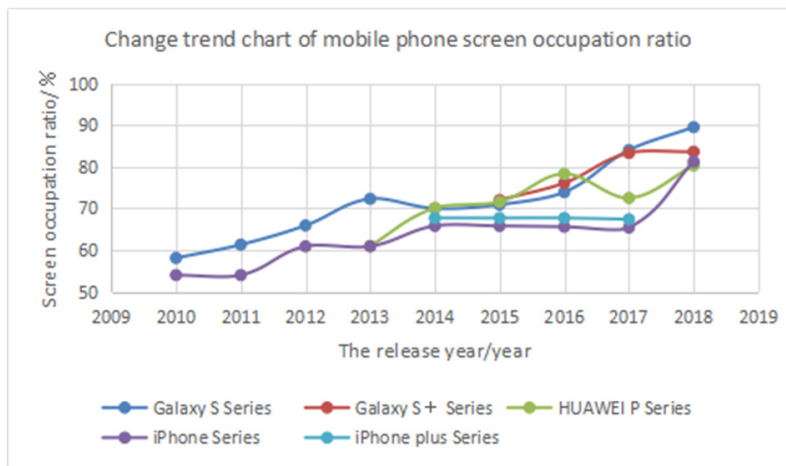

Figure 2. Change trend chart of mobile phone screen occupation ratio

According to the Figure 2, it can be seen that since 2010, the proportion of mobile phone screens of each brand series has been steadily rising, except for the iPhone series and iPhone plus series which have no change in screen occupation ratio and screen size from 2014 to 2017. In addition, the percentage of other mobile phone screens has gradually increased in the past nine years.

According to the above analysis, the mobile phone screen size cannot be infinitely large, but in order to further increase the utilization rate of the frontal area of the mobile phone and enhance the user experience, all major manufacturers have chosen to develop and introduce products with a higher proportion of screens 
and enhance brand competitiveness. Since the mobile phone products have been developed relatively well so far, we have continuously increased the proportion of screens, developed full-screen technology, and met the development trend of the mobile phone market to meet the specific needs of mobile phone users.

\subsection{The negative effect caused by the oversized cell phone screen}

Although mobile phone with large-screen enhances the user's visual experience, it brings many inconveniences and is harmful to people. This article will propose three negative effects that the screen is too large to users after investigation and study, then analyze the reason why the screen size of the mobile phone tends to be stable, finally infer the optimal screen size value.

The first impact: Excessive screen size is harmful to human health

While large-screen mobile phones provide users with more information, they gradually make it difficult to operate the screen with one hand. According to the physiological structure of the human hand, the main activity finger in the single-handed operation of the mobile phone is the thumb, but due to the limitation of the knuckle length, the reach of the thumb is very limited, so long-term single-handed operation of the large-screen mobile phone may cause hand joints, muscle and ligament injuries ${ }^{[7]}$. If operators use both hands for a long time, fatigue of the hand muscles and inflexible hand movements may be caused, causing stenosis tenosynovitis ${ }^{[8]}$. In addition, due to the small viewing distance when using a mobile phone, excessive screens cause eye fatigue and decreased vision.

The second impact: Excessive screen size is not convenient for daily carrying.

According to people's habits, especially for male users, it is more convenient to carry a mobile phone into a pocket of clothes every day to conveniently view information at any time. However, as mobile phone screens become larger and larger, this type of carrying method will inevitably cause inconveniences. Especially when the clothes are thin in summer, the large-screen mobile phones are difficult to be fully loaded into pockets, which not only makes the user feel uncomfortable, but also can easily fall or be stolen. It can be seen that the screen is too large to a certain extent, does not meet the requirements of humanized design.

The third impact: Excessive screen size increases screen breakage.

As the screen of smart phones continues to develop toward the full-display, the chance of screen breakage increases as the proportion of screens increases. The damage of the screen will greatly reduce the user's satisfaction with the product, and replace the screen will also cost a lot of money. Therefore, the reinforcement of the screen will also become the focus of future research for major manufacturers. However, the screen is fragile and the service life reduction is the key negative effect caused by the large screen size.

\subsection{Suggestions}

Through the analysis of the screen size and screen occupation ratio of the mobile phone, and the summary of the negative impact on the screen, it can be concluded that the screen size should be within a benign range, while the screen occupation ratio can be continuously improved with the progress of technology.

According to the law analysis and questionnaire survey, the best values for the screen size are 4.7 inches, 5.5 inches and 5.1 inches. Therefore, this study can conclude that the best range of screen size is 4.7-5.5 inches. Mobile phone manufacturers can make different choices according to the brand development status. In addition, the questionnaire survey found that the difference between male and female needs is not significant, but there are still some differences. Therefore, mobile phone manufacturers can launch a series of targeted products by dividing the user population. For example, launching iPhone and iPhone plus with different screen sizes is an excellent strategy.

\section{Conclusion}

This study summarizes the development status of mobile phone screens of Samsung, Apple, and HUAWEI in recent years. Based on the user experience, this paper summarizes the best screen size and the development direction of the screen, which can provide theoretical support for manufacturers to design mobile phone, help them adjust the direction of research and development, and develop new product development strategies.

At the same time, there are still some shortcomings in this study. For example, the purpose of this paper is to analyze only part of the information in the form, the other parts still have high research value, and may have a little impact on the results of the study. Secondly, the questionnaire survey content is single and the number of samples is less. Due to the large number of mobile phone users, 160 valid questionnaires are not sufficient to accurately represent all users. In light of these deficiencies, this research will continue in the future to obtain more accurate and complete research results.

\section{References}

1. Hassenzahl M. International Conference on Association Francophone d'Interaction Homme -Machine, 339, 11(2008)

2. Si Yaqing, Liu Lei, Software, 3, 111(2015)

3. Tian Meiling, Zhao Xinjun, Science Innovation and Brand, 6, 52(2013)

4. Wang Songpei, Wang Wei, Xu Shanshan, Zhang Qi, Enterprise Guide, 3, 44(2015)

5. Xin Yizhong, Li Yang, Li Yan, Jiang Xinhui, Journal of Computer-Aided Design \& Computer Graphics, 28, 1750 (2016)

6. Karlson A, Bederson B, Contreras J, USA:IGI Global Press, 86(2006) 
7. YS Park, SH Han, International Journal of Industrial Ergonomics, 1, 68 (2010)

8. Zhu Shenbo, Cui Lanhai, Chen Zhengnan, Wu Jiaqian,
Shen Zhongzhou, Zhang Jiyong, Health Medicine Research and Practice, 13, 24 (2016) 